\title{
CNS-Immune Reconstitution Inflammatory Syndrome in the Setting of HIV Infection, Part 2: Discussion of Neuro-Immune Reconstitution Inflammatory Syndrome with and without Other Pathogens
}

\author{
M.J.D. Post, M.M. Thurnher, D.B. Clifford, A. Nath, R.G. Gonzalez, R.K. Gupta, and K.K. Post
}

\begin{abstract}
SUMMARY: While the previous review of CNS-IRIS in the HIV-infected patient on highly active antiretroviral therapy (Part 1) dealt with an overview of the biology, pathology, and neurologic presentation of this condition and a discussion of the atypical imaging findings in PML-IRIS and cryptococcal meningitis-IRIS due to the robust inflammatory response, the current review (Part 2) discusses the imaging findings in other commonly encountered organisms seen in association with CNS-IRIS, namely, VZV, CMV, HIV, Candida organisms, Mycobacterium tuberculosis, and Toxoplasma gondii. Also described is the imaging appearance of CNS-IRIS when not associated with a particular organism. Recognition of these imaging findings will give credence to the diagnosis of CNS-IRIS and will allow the clinician to institute changes in medical management, if necessary, so that immune reconstitution and improved patient outcome can occur with time.
\end{abstract}

ABBREVIATIONS: $\mathrm{CMV}=$ cytomegalovirus; HAART = highly active antiretroviral therapy; HIVE = HIV encephalitis; $\operatorname{lgG}=$ immunoglobulin G; IRIS = immune reconstitution inflammatory syndrome; $\mathrm{MAC}=$ Mycobacterium avium complex; $\mathrm{PCR}=$ polymerase chain reaction; $\mathrm{PML}$ = progressive multifocal leukoencephalopathy; $\mathrm{TB}=$ tuberculosis; $\mathrm{VZV}=$ Varicella zoster

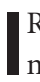

RIS is a complex and a not-yet completely well-understood phenomenon that is seen most commonly in the setting of HIV infection, ${ }^{1-46}$ a setting that is the focus of this review, which highlights CNS-IRIS. The complexity of this condition is due to the significant changes in biology that take place in the setting of immune reconstitution following the initiation of HAART as well as to the varying and often atypical clinical and imaging expressions of this syndrome. ${ }^{1-46}$ While an increased inflammatory pathology is the hallmark of this condition with CD8+ T-cell lymphocytic infiltration overshadowing other pathologic changes, often biopsy or postmortem material is not available to confirm the presence of IRIS. 1,16,23,24 If pathologic criteria cannot be used, then in addition to difficulties in exactly defining IRIS, there are also challenges to the diagnosis of this condition. For example, the

From the Section of Neuroradiology (M.J.D.P.), Department of Radiology, University of Miami Miller School of Medicine, Jackson Memorial Medical Center, Miami, Florida; Department of Radiology (M.M.T.), University of Vienna, University Hospital Vienna, Vienna, Austria; Department of Neurology (D.B.C.), Washington University in St. Louis, St. Louis, Missouri; Section of Infections of the Nervous System (A.N.), National Institute of Neurological Disorders and Stroke, National Institutes of Health, Bethesda, Maryland; Department of Radiology (R.G.G.), Harvard Medical School and Massachusetts General Hospital, Boston, Massachusetts; Department of Radiology (R.K.G.), Sanjay Gandhi Postgraduate Institute of Medical Sciences, Lucknow, India; and Department of Internal Medicine (K.K.P.), UMass Memorial Medical Center-University Campus, Worcester, Massachusetts.

Please address correspondence to M. Judith Donovan Post, MD, Section of Neuroradiology, Department of Radiology, University of Miami Miller School of Medicine, Jackson Memorial Medical Center, West Wing 279, 1611 NW 12th Ave, Miami, FL 33136; e-mail: jpost@med.miami.edu

- Indicates open access to non-subscribers at www.ajnr.org

http://dx.doi.org/10.3174/ajnr.A3184 clinical scenario seen in association with this robust and often overzealous inflammatory response may vary from very mild to fulminating, thereby complicating the diagnosis of IRIS. ${ }^{1}$ Furthermore, even imaging findings may be diverse. While often atypical in appearance from the pre-HAART era, as exemplified by PML-IRIS and cryptococcal meningitis-IRIS, the imaging findings in toxoplasma encephalitis-IRIS and Mycobacteria tuberculosis-IRIS may not show any dramatic changes from those seen in HIV-infected patients before HAART initiation. Nevertheless, whether or not the MR imaging or CT appearance is strikingly different, increasing parenchymal high FLAIR signal abnormalities, contrast enhancement of the leptomeningeal spaces and/or of the parenchymal lesions, mass effect, and restricted diffusion still appear to be features that predominate in CNS-IRIS. It is these imaging findings, then, that are a direct result of the inflammatory response that has been evoked by HAART, along with a worsening or change in neurologic symptoms or signs, which should raise the suspicion for CNS-IRIS. Once recognized, the clinician may opt to add specific therapy, such as steroids, to overcome a toorobust inflammatory reaction, which will then allow time for immune restoration and eventual improved patient outcome to occur. The object of this review, then, is to examine the imaging characteristics in CNS-IRIS that have been reported in viruses other than the papovavirus, in fungi other than Cryptococcus organisms, in mycobacteria, and in parasites as well as in cases without organisms so that this condition can be recognized and treated when necessary. 


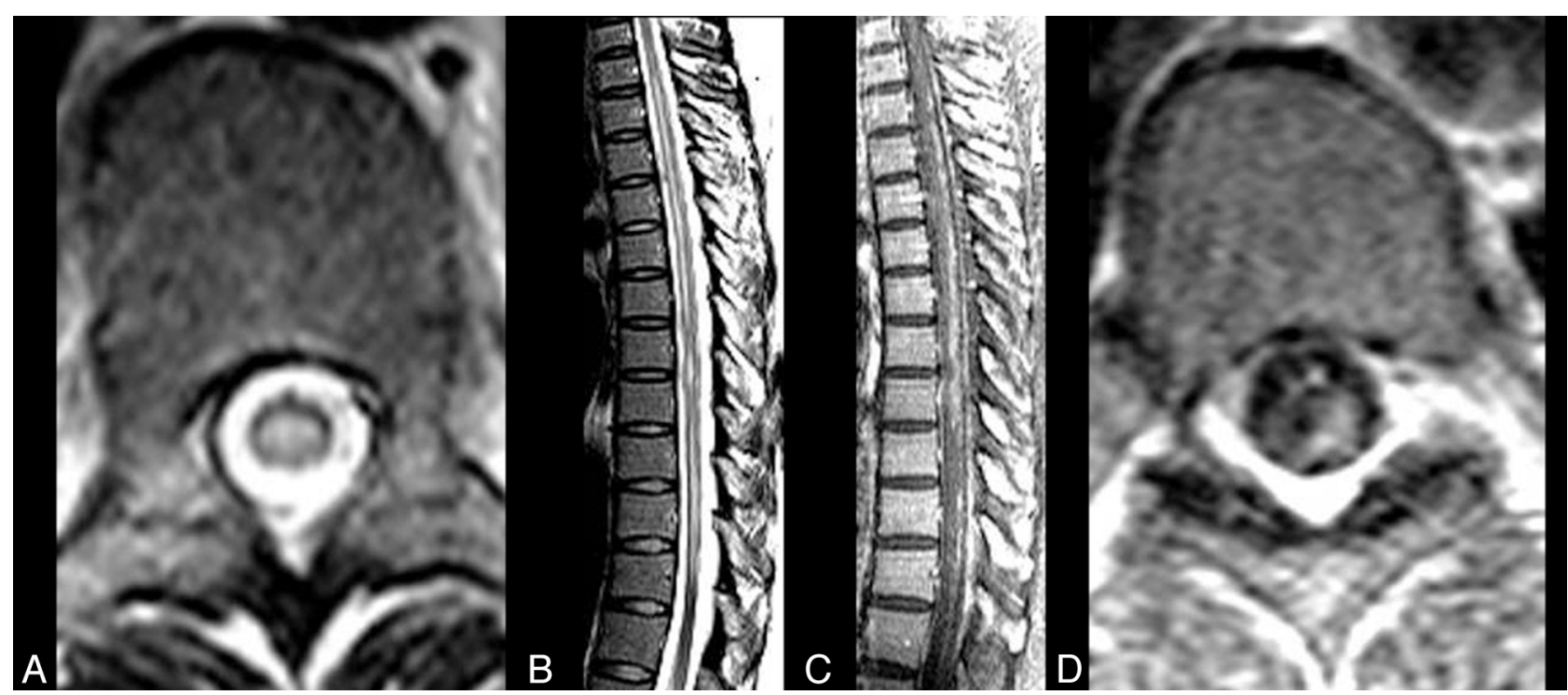

FIG 1. CNS VZV-IRIS. A 37-year-old HIV + woman with a herpes zoster rash with a 77 dermatome was treated with acyclovir 6 weeks prior to admission (PTA). Four weeks PTA, the patient developed leg numbness and tingling. HAART was initiated 2 weeks PTA. Double vision was followed by severe weakness in her legs 1-2 days PTA. Initial MR imaging of the thoracic spine with fast spin-echo axial $(A)$ and sagittal $(B)$ views shows scattered patchy extensive high-signal lesions in the spinal cord, which enhance on contrast sagittal (C) and axial (D) contrast TIWI and are associated with leptomeningeal enhancement, indicating an inflammatory VZV meningitis and myelitis.

\section{CNS-IRIS WITH ORGANISMS \\ Virus}

VZV CNS-IRIS. VZV vasculopathy affecting either large or small vessels leading to infarcts in the brain has been reported in the setting of CNS-IRIS, but it is said to be rare. ${ }^{4-50}$ In a case report by Newsome and Nath ${ }^{47}$ of VZV-IRIS, MR imaging showed nonenhancing brain stem lesions associated with supratentorial white matter lesions, which were later associated with new thalamic and subcortical infarcts as the patient worsened on HAART, concomitant with a rising CD4 count and a markedly decreasing HIV viral load. High-dose intravenous corticosteroids and tapered oral prednisone eventually resulted in significant improvement in this patient with VZV vasculopathy. ${ }^{47}$

In another investigation by Nagel et $\mathrm{al}^{48} 30$ patients with VZV vasculopathy were studied, 11 of whom were immunocompromised (5 with HIV or AIDS). While not focusing on IRIS, the authors did provide an analysis of the typical clinical, laboratory, and imaging findings in CNS VZV vasculopathy. These authors found that for the diagnosis of VZV vasculopathy, there was a greater sensitivity for the detection of VZV IgG in the CSF (93.33\%) than for the PCR detection of VZV DNA in the CSF $(30 \%) .{ }^{48}$ This detection of the IgG antibody was even higher in the immunocompromised population (100\%) as was the detection of CSF pleocytosis (82\% versus 58\%). ${ }^{60}$ The rash typical of herpes zoster was evident in only $54 \%$ of the immunosuppressed patients versus $68 \%$ in those with an intact immune system. ${ }^{48}$ The lack of a rash in many patients and the long time from the development of the rash to the onset of neurologic symptoms (4.1 months average interval) in both groups led the authors to suggest that if a TIA or stroke occurs in a patient, VZV vasculopathy or viral infection in cerebral arteries (with Cowdry type A inclusion bodies, herpesvirus particles, and multinucleated giant cells) must be entertained even in the absence of the typical rash. ${ }^{48}$

Interestingly enough, it was the finding of an infarct on MR imaging or CT that was actually the most consistent clue indicating the diagnosis of VZV vasculopathy in those patients with a positive CSF PCR or VZV IgG. Negative findings on conventional angiography or MRA did not exclude the diagnosis of VZV vasculopathy because the type of vasculopathy revealed on imaging studies in both groups (focal vascular stenosis or occlusion) was not restricted to large-vessel disease because small-vessel disease (affecting the perforating arteries) and a mixed large- and smallvessel disease pattern could also be seen. ${ }^{48}$ In fact, $30 \%$ of both groups had either negative findings on conventional angiography or MRA because small-vessel involvement went undetected on conventional angiography and MRA.

Large-artery involvement was actually only seen in $13 \%$ of the combined cohort; small artery involvement, in 37\%; and a mixed pattern, in $50 \%{ }^{48}$ In contrast, $97 \%$ of patients had positive findings on MR imaging or CT, making the diagnosis of VZV vasculopathy very unlikely in the presence of negative findings on MR imaging or CT. ${ }^{48}$ Restricted diffusion in acute infarcts, often multifocal and often affecting the white matter or the gray-white matter junction, could be seen as well as white matter demyelination. ${ }^{48}$ The thalamus, hypothalamus, and posterior limb of the internal capsule were sites of infarction as well. ${ }^{48}$ However, no mention of enhancement was made, suggesting that enhancement was not a typical imaging feature in these patients without IRIS.

In contrast, in the 2 patients with VZV-IRIS illustrated in Figs $1-4$, imaging clues to the diagnosis of VZV-IRIS included leptomeningeal enhancement in the spinal subarachnoid spaces as well as patchy spinal cord enhancement at sites of high T2 signal intensity along with intracranial leptomeningeal enhancement and patchy peripheral high FLAIR signal and enhancement in the adjacent parenchyma along with vasculitis and infarcts. The avid enhancement was likely due to the robust inflammatory response caused by IRIS. While retrospective review revealed that both of 


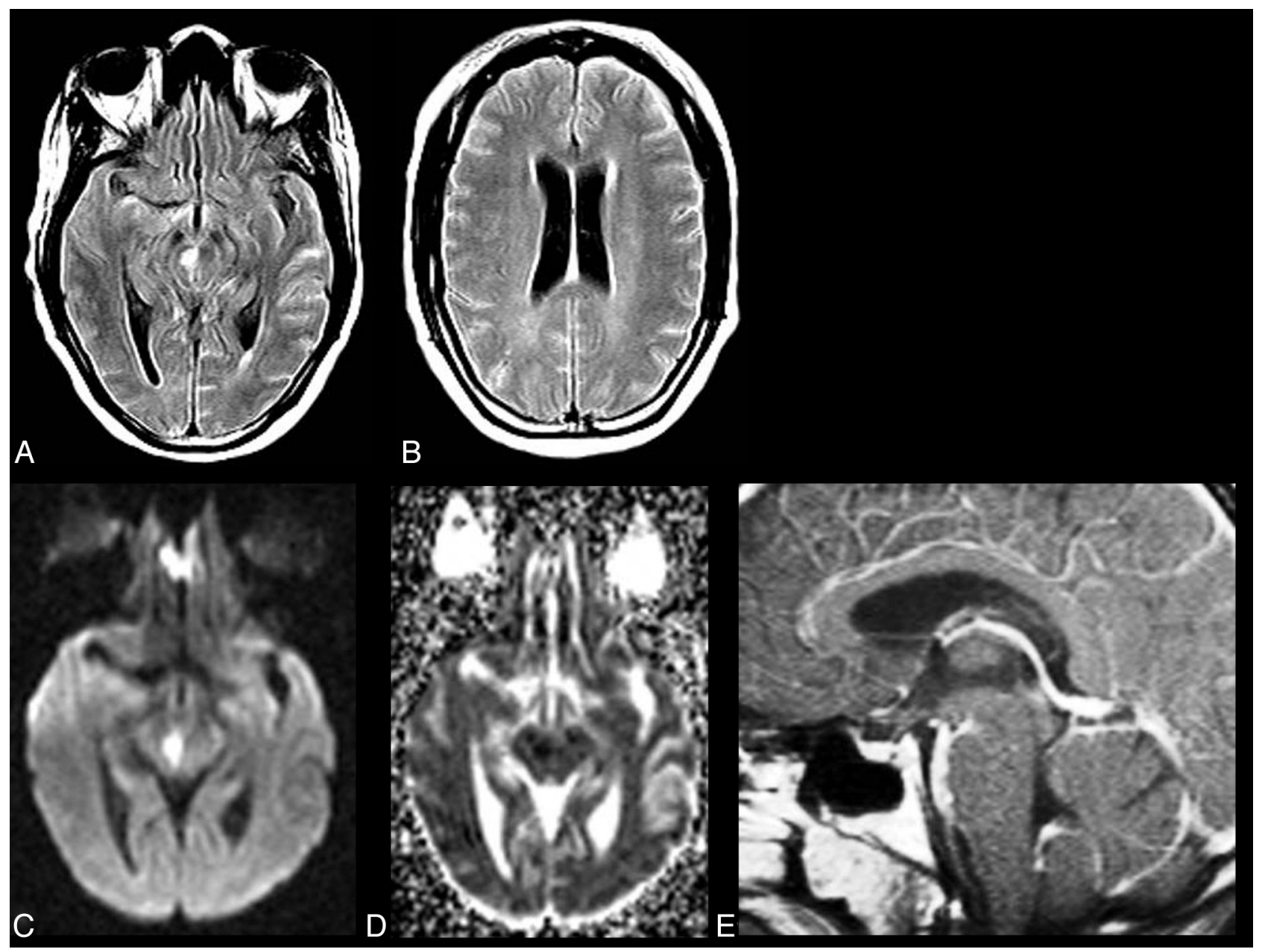

FIG 2. CNS VZV-IRIS (same patient as in Fig 1). When the patient developed a right third-nerve palsy and a left hemiparesis, initial brain MR imaging shows, on axial FLAIR ( $A$ and $B$ ), hyperintense signal in the subarachnoid spaces diffusely compatible with meningitis and focal high signal in the right midbrain along with a mild communicating hydrocephalus. Restricted diffusion is demonstrated in the midbrain on DWI (C) and ADC maps (D), indicative of an acute infarct. Contrast TIWI in sagittal projection (E) reveals leptomeningeal enhancement in the interpeduncular fossa and prepontine cisterns. CSF PCR was positive for varicella zoster virus. Her CD4 count was $140 \mathrm{cells} / \mu \mathrm{L}$. Intravenous acyclovir was started and HAART was stopped. Three weeks later progression to paraplegia and right upper extremity weakness occurred, several days after completion of the acyclovir therapy.

these patients did indeed have a dermatomal rash many weeks earlier, this clinical clue may be absent in some patients presenting with VZV-IRIS. Therefore, the neuroradiologist may play an important role by suggesting the diagnosis of VZV-IRIS when the MR imaging clues of enhancement of the leptomeningeal spaces, spinal cord, and brain parenchyma (which is not typical in patients without IRIS), vascular beading, and infarcts are seen. The neuroradiologist may then be the first to suggest this diagnosis of VZV-IRIS, alerting the clinicians to confirm the diagnosis with a positive CSF test. ${ }^{49}$

CMV-IRIS: Vitritis and Encephalitis. Among the organs that can become the target of an abnormal immune response due to the $\mathrm{CD} 8+$ dysfunction triggered by HAART is the eye. ${ }^{51}$ In a study by Karavella et al, ${ }^{52} 63 \%$ of patients with CMV retinitis and HAART developed an "immune recovery virtritis" after 10 months. The CMV retinitis was inactive, though this vitritis can also occur in patients with active retinitis as well. ${ }^{32,52}$ Jacobsen et $\mathrm{al}^{53}$ also reported a transient inflammatory reaction in the vitreous in patients with AIDS with CMV retinitis on antiretroviral therapy.
Residual CMV antigens or proteins are thought to be the antigenic stimulus for this IRIS phenomenon. ${ }^{25}$

In addition to the eye and the spine (where a CMV radiculitis has been reported in the presence of CMV infection, HIV, and HAART), another organ that can be targeted by the over-reactive immune system is, of course, the brain. ${ }^{30}$ In an HIV + patient noncompliant to antiretroviral therapy and to treatment for CMV colitis, CMV encephalitis developed, characterized by both typical and atypical imaging features. ${ }^{54}$ Instead of the more typical MR imaging findings of ventriculitis or even solitary focal mass lesions, this patient, who died from his CMV encephalitis, had MR imaging showing widespread multifocal areas of restricted diffusion and faint solid or peripheral enhancement in both the supraand infratentorial compartments. ${ }^{54}$ Periventricular and corpus callosum white matter was involved as well as subcortical white matter and the basal ganglia, brain stem, and cerebellum. ${ }^{54}$ The largest lesion in the precentral gyrus had mass effect and edema. Restricted diffusion correlated at postmortem examination with areas of necrosis, calcifications, and large CMV inclusions and 


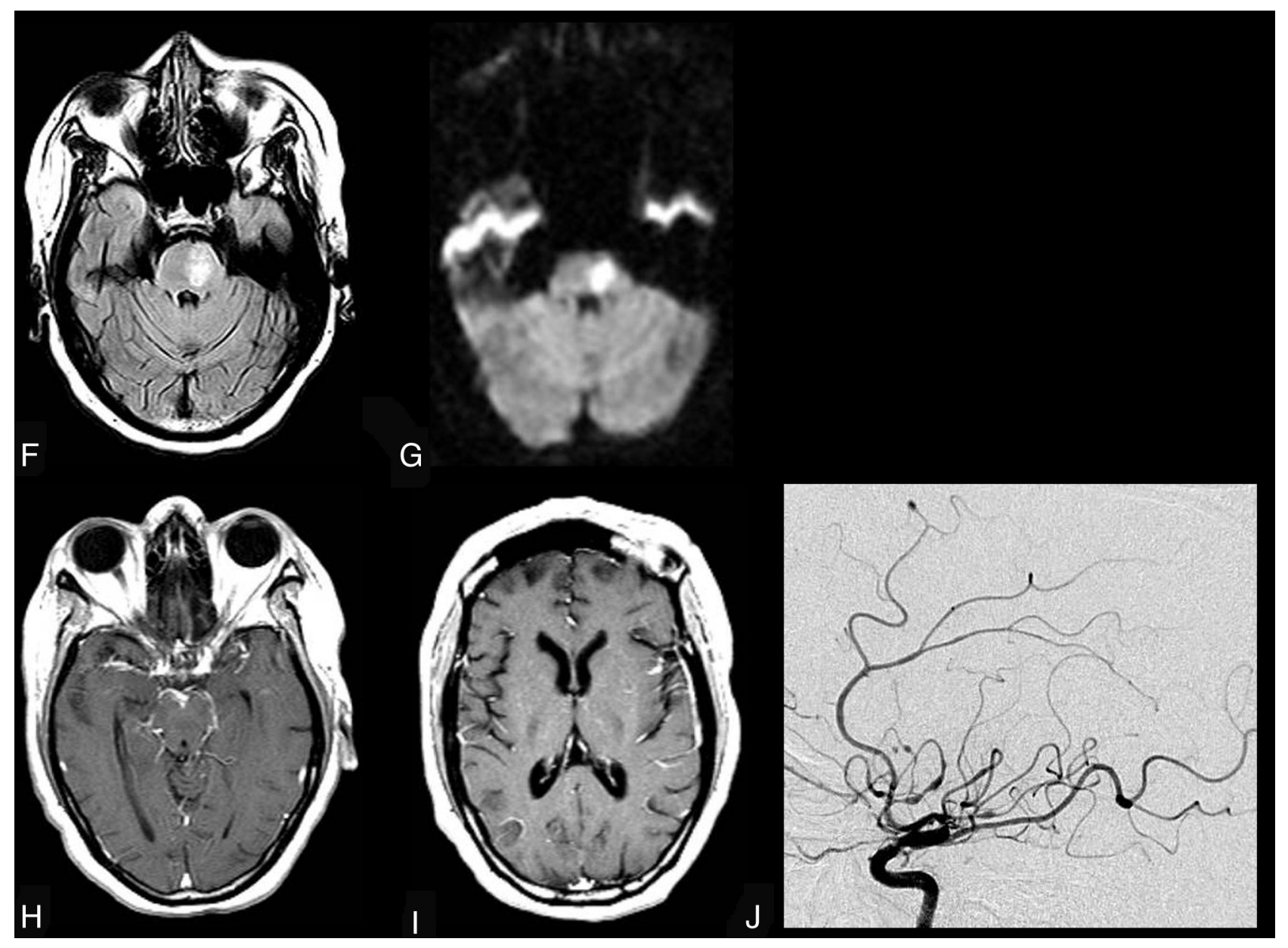

FIG 2 (CONTINUED). CNS VZV-IRIS (same patient as in Fig. 1). Follow-up MR imaging shows new high signal on FLAIR $(F)$ in the pons along with restricted diffusion on DWI (G) and ADC maps (latter not shown), compatible with another acute infarct. Contrast TTWI in axial (H and I) views reveals leptomeningeal enhancement along with focal enhancement at multiple sites, including in the left Sylvian fissure, which conventional angiography demonstrates is due to a vasculitis with vessel beading, narrowing, and aneurysms (l) as seen on lateral view of the left carotid injection. Acyclovir was restarted, and steroids were added.

was thought to be related to cytotoxic edema from cell death. ${ }^{54}$ While it is not directly linked by the authors to CMV-IRIS, we suspect that IRIS may have played a role in this patient's atypical presentation because the patient had been treated with HAART but had adherence issues. ${ }^{54}$

In another report, an HIV + patient developed visual changes 2 weeks after the initiation of HAART attributed to CMV retinitis for which he was treated with anti-CMV therapy. ${ }^{55}$ Two weeks thereafter, he developed neurologic changes with rising CD4 counts and falling plasma HIV RNA levels as well as an active vitritis related to CMV-IRIS. ${ }^{55}$ On MR imaging, areas of high FLAIR signal and restricted diffusion were found in the brain stem, basal ganglia, periventricular white matter, and internal capsule. $^{55}$ These findings were postulated as being due to acute and subacute infarcts from a vasculitis in the territories of small vessels due to the infection by CMV of the endothelial cells in the brain vasculature, as supported by postmortem studies reported in the literature showing CMV inclusions in capillary endothelium as well as in astrocytes and neurons. ${ }^{55,56}$ Therapy with intravenous ganciclovir and foscarnet resulted in clinical and MR imaging improvement, with evolution on MR imaging of the presumed infarctions. ${ }^{55}$ Enhancement not seen initially appeared after 2 weeks of medical therapy and was attributed to the presence of subacute enhancing infarcts. ${ }^{55}$

The vasculitis and infarcts proposed with both CMV and varicella zoster-IRIS can be mimicked on MR imaging by primary cerebral angiitis related to IRIS. In those rare cases, a vasculitis unrelated to an infectious agent is responsible for the infarcts and vessel beading, with segmental vessel narrowing seen on MR imaging and MRA. This primary cerebral angiitis is thought to be related to an inflammatory process in HIV + patients on HAART with low plasma HIV RNA levels. No organisms are found, and the patients may respond to corticosteroid therapy. ${ }^{57}$

HIVE-IRIS. While temporary progression of high T2/FLAIR periventricular white matter abnormalities have been reported on MR images in 4 patients with AIDS dementia complex shortly after the initiation of HAART with regression or stabilization of these MR imaging white matter lesions with time, these patients did not have neurologic deterioration and actually were clinically improved at the time of the MR imaging worsening. ${ }^{58}$ A breakdown of the blood-brain barrier with a temporary increase in water content in 3 of the 4 patients was thought to be the cause of the increased white matter signal abnormalities on MR imaging. ${ }^{58}$ 


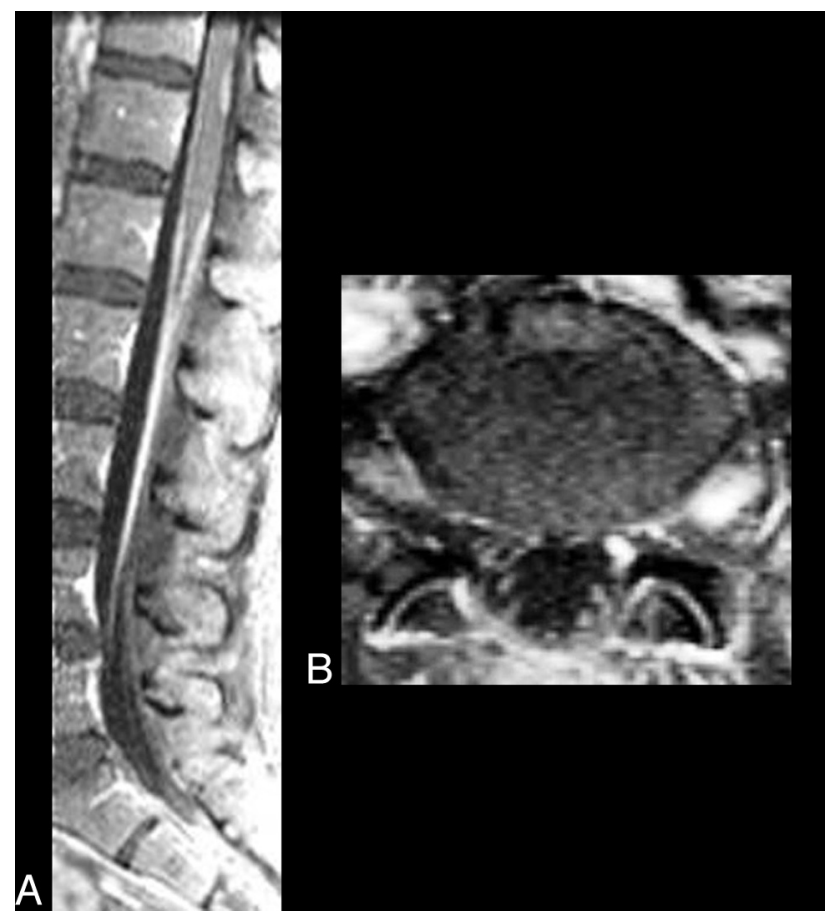

FIG 3. CNS VZV-IRIS. A 51-year-old woman who, 3 weeks prior to admission at an outside hospital, was newly diagnosed with seizures and HIV and was started on HAART. She was also treated with antibiotics for a leg cellulitis. Altered mental status and tonic-clonic seizure prompted a new hospital admission 3 weeks later where herpes zoster lesions of her left foot were seen, and acyclovir was begun. Absolute CD4 count was 283 cells/ $\mu \mathrm{L}$. CSF cultures and PCR were negative. Fat-saturated contrast sagittal $(A)$ and axial $(B)$ images show enhancement of the entire course of the left S1 nerve root, compatible with a radiculitis, and enhancement and enlargement of the left dorsal root ganglia. Some thoracolumbar leptomeningeal enhancement is also seen indicating meningitis.

In contrast, HIV encephalitis-IRIS with clinical worsening is a rare occurrence. ${ }^{19}$ Only a handful of cases have been described in which patients with HIV dementia sustained an acute neurologic deterioration following the institution of HAART. ${ }^{19}$ Two patients died only 1 and 3 months after HAART initiation. ${ }^{59}$ T-cell lymphocytic infiltration was found at postmortem examination in the perivascular spaces, and lymphocytes and macrophages were found in the white and gray matter and in the leptomeninges. ${ }^{19}$ Immunohistochemistry showed staining of the T-cells in the parenchyma and around the blood vessels for cytotoxic granules. ${ }^{19,59}$ Because the cytotoxic granules are known to contain enzymes such as granzyme B, it has been postulated that interaction with a G-protein-coupled receptor can lead to neurotoxicity. ${ }^{19}$ MR imaging was instrumental in showing white matter progression during a 1-year time period in another HIV + patient with accelerating dementia whose biopsy demonstrated CD8+ T-cells in perivascular and parenchymal infiltrates and around neurons. ${ }^{19}$ This patient had a transient response to steroids. Langford et $\mathrm{al}^{60}$ reported 7 more cases thought to be related to HIVIRIS, in which perivascular inflammatory cells were found with lymphomonocytic exudates, myelin loss, and axonal injury with astrocytic gliosis.

Yet another entity recently mentioned has been an HIV-related acute inflammatory leukoencephalopathy of undetermined origin, in which an inflammatory reaction has characterized the
MR imaging and histologic findings and may also be related to immune-mediated reactions of HIV in the brain. ${ }^{61}$

\section{Fungus}

Candida Meningoencephalitis-IRIS. In a case report by Berkeley et $\mathrm{al}^{16}{ }^{16}$ the challenges encountered in instituting HAART in those with unrecognized opportunistic infections was made clear as was the difficulty in diagnosing meningitides due to certain fungi. In their case, a patient with AIDS presenting with a subacute meningitis for which no organism was identified was given antituberculous medications and restarted on HAART, with a subsequent decrease in viral load though no rise in the CD4 count. ${ }^{16}$ Neurologic deterioration rapidly ensued, and the patient soon died. MR imaging a short time before his death showed new high-signal abnormalities on FLAIR in the brain stem and thalamus. ${ }^{16}$ (No reference was made to the use of contrast or diffusion imaging.) Autopsy revealed pathology due to IRIS with a meningitis due to Candida organisms as well as a vasculitis related to CD8+ T-cell lymphocytic infiltration. ${ }^{16}$ Inflammatory changes in the basilar meninges were accompanied by extensive destruction of all the wall layers of the basilar artery by the inflammatory process with a predominance of lymphocytes along with some plasma cells and multinucleated giant cells. ${ }^{16}$ Numerous microinfarcts were found in the brain stem with vacuolization. ${ }^{16}$ Candida organisms were identified in the meninges on special stains, but only a few organisms were found in the brain stem parenchyma or basilar artery. ${ }^{16}$ Immunostaining revealed that the inflammatory cells were almost exclusively CD8 + lymphocytic cells, which were found not only in the meninges but also in the walls of the vertebral-basilar circulation as well as scattered throughout the brain stem parenchyma. ${ }^{16}$ In contrast to candida meningitis in the pre-AIDS era when an inflammatory reaction did not play a significant role, in this HIV + patient with chronic candida meningitis initiated on HAART, the inflammatory process or IRIS was paramount and led to a severe vasculitis with secondary brain stem infarction, the cause of the patient's neurologic decline. ${ }^{16}$ Because the high mortality rates in candida meningitis have been reported to be reduced with treatment to $10 \%-30 \%$, diagnosis of this infection, which can simulate on CSF profile TB meningitis, is critical so that treatment can be initiated, especially before beginning HAART. ${ }^{16}$

\section{Mycobacteria}

Atypical Mycobacterial CNS-IRIS. As the most commonly occurring bacterial infection in patients with AIDS, MAC would be expected to commonly involve the brain. ${ }^{62}$ However, brain involvement by MAC is actually rare but, when present, is characterized by a granulomatous inflammation and by lymphocytes and macrophages aggregated in a perivascular location. ${ }^{62,63}$ Also rarely occurring is MAC-related CNS-IRIS despite the fact that MAC is frequently identified as a pathogen causing IRIS outside the CNS with pulmonary disease and lymphadenitis, and despite the fact that the phenomenon of IRIS was first described in an $\mathrm{HIV}+$ patient who developed MAC infection following antiretroviral therapy. ${ }^{1,19,62-64}$ In a clinicopathologic description, Kishida and Ajisawa ${ }^{62}$ reported one such unusual case in an $\mathrm{HIV}+$ patient with disseminated MAC, who had multiple en- 


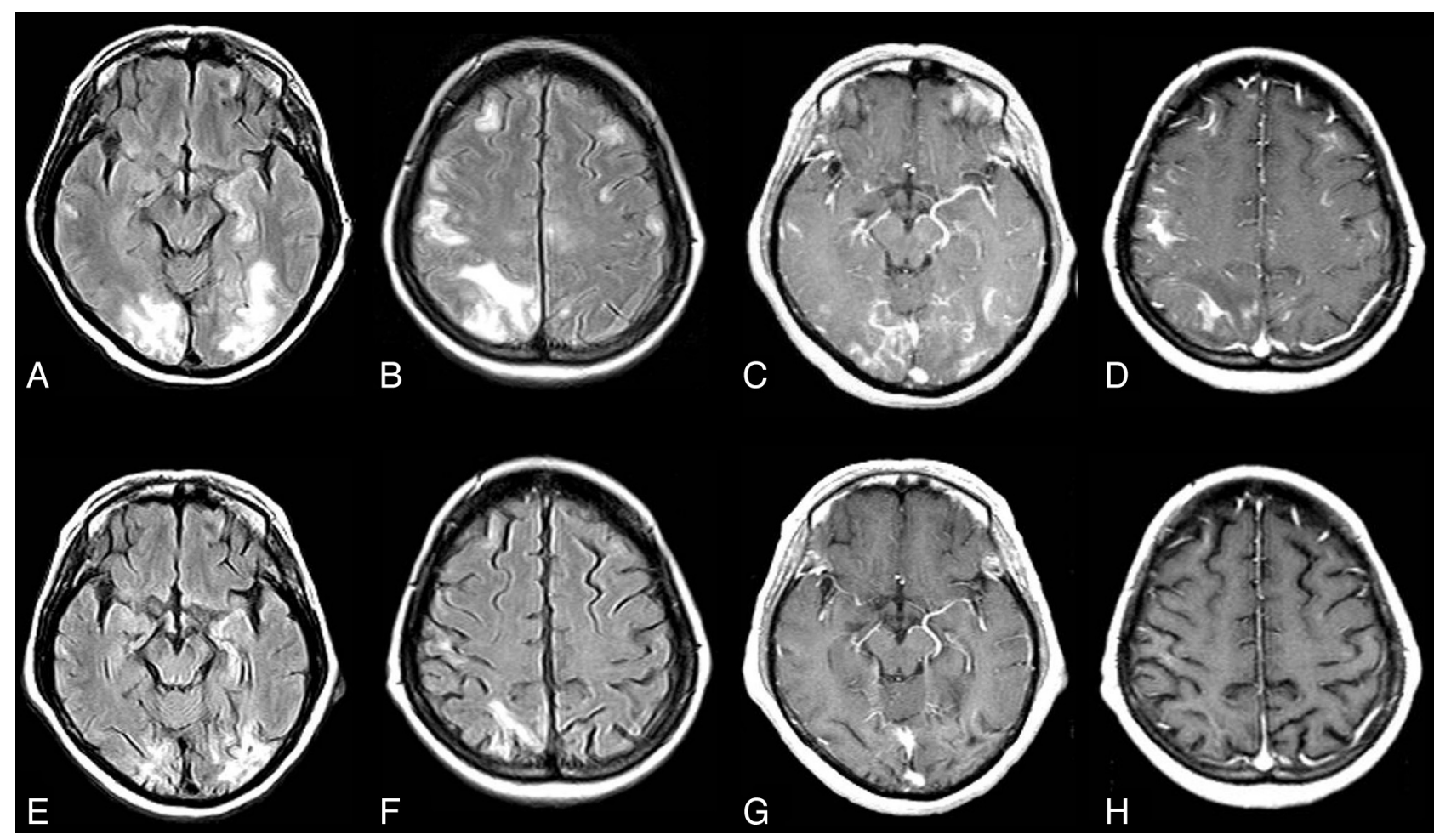

FIG 4. CNS VZV-IRIS (same patient as in Fig 3). Initial MR imaging of the brain shows, on axial FLAIR $(A$ and $B)$ and contrast TTWI ( $C$ and $D)$, bilateral hyperintense signal abnormalities without restricted diffusion in the cortical and subcortical regions of the occipital, temporal, frontal, and parietal lobes with leptomeningeal and some patchy adjacent parenchymal enhancement with mild sulcal compression and no MRA (not shown) abnormalities. She was restarted on HAART and finished a 3-week course of acyclovir followed by suppressive therapy. Follow-up MR imaging 1 year later $(E-H)$ shows a marked decrease in FLAIR $(E$ and $F)$ high-signal abnormalities with concomitant atrophy and near resolution of the leptomeningeal and parenchymal areas of enhancement, shown on axial contrast images ( $G$ and $H)$.

hancing lesions in the brain. Follow-up MR imaging showed treatment response to MAC. HAART was then instituted (6 months after the original diagnosis of MAC) ${ }^{62}$ The patient then developed fever (1 month after initiation of HAART), cavitary lesions in the lungs (after 2.5 months), and new intracranial lesions on MR imaging (after 4 months). A multilobulated ringenhancing mass adjacent to the left temporal horn in the left temporal lobe with considerable surrounding edema was seen along with subependymal enhancement of the lateral ventricles. ${ }^{62} \mathrm{Au}-$ topsy in this patient, who rapidly died, revealed a granulomatous reaction in the wall of the temporal lobe mass with fibrous tissue and lymphocytes and a central area of necrosis comprised of pleomorphic cells. ${ }^{62}$ Collagen, epitheloid cells, lymphocytes, and multinucleated giant cells were seen in the periventricular region without detection of a residual organism. ${ }^{62}$ These lesions in the brain were thought to be related to an overexuberant pathogenspecific inflammatory response to the dead or dying organism or to the antigen to MAC. ${ }^{62}$ Tissue destruction ensued because of this exaggerated immune response. ${ }^{62}$

Mycobacterium Tuberculosis. Just as clinical worsening has been known to occur in the HIV-negative patient with TB following antituberculous therapy because of a heightened pathologic inflammatory response to improved immune function, ${ }^{19}$ clinical deterioration can also occur, especially systemically, in HIV + patients with TB when HAART is initiated. IRIS occurs in approximately $16 \%$ of patients with AIDS with TB and antiretroviral therapy, of whom $3 \%$ die. $^{30}$ In fact, TB is reported to be the infection most commonly associated with IRIS extracranially ${ }^{8}$ and usually occurs within 2 months of antiretroviral therapy when not involving the CNS. ${ }^{25}$ In HIV + patients with $M$ tuberculosis whose antituberculous therapy was started within 1-6 months before initiation of HAART, 43\% developed extracranial TB-IRIS at 2-114 days (12-day median) after HAART, manifested systemically by lymphadenopathy, fever recurrence, skin lesions, worsening lung infiltrates, and gastrointestinal symptoms. ${ }^{65}$ TB-IRIS occurred more often in patients with lower CD4 counts and higher HIV-1 RNA levels at baseline and more often in those with extrapulmonary $\mathrm{TB} .{ }^{65}$ Cultures in the sputum and lymph nodes were negative, and patients improved after the addition of anti-inflammatory medication. ${ }^{32}$ In yet another study, an increase in the first month of antiretroviral therapy of the CD4 percentage of $\geq 12 \%$ and a rapid rise in the $\mathrm{CD} 4 / \mathrm{CD} 8$ ratio of $>33 \%$ were identified as risk factors with the highest predictive values for developing TBIRIS in HIV+ individuals. ${ }^{8}$

As for TB-associated CNS-IRIS, the incidence is said to be low. ${ }^{19}$ In a study by Pepper et al, ${ }^{66}$ it was reported in $12 \%$ of 190 patients with paradoxical TB, a condition in which antitubercular treatment precedes antiretroviral therapy. Of these 23 patients with neurologic TB-IRIS and coinfection with HIV-1, meningitis was found in 8; tuberculoma, in 7; meningitis and tuberculoma, in 5; and radiculomyelopathy, in $3 .^{66}$ Corticosteroids were administered in $91 \%$. Considering the 19 of 23 patients in whom corticosteroids were added to the antitubercular therapy, $18 \mathrm{dem}$ onstrated initial improvement. ${ }^{66}$ At 6 months, the death rate was 
$3 \%$. CT was used to detect the tuberculoma that was seen in 1 patient 16 days after antiretroviral initiation and the onset of headache, stiff neck, and vomiting; the tuberculoma was a $1-\mathrm{cm}$ inhomogeneous lesion with surrounding edema in the left temporal-parietal region. ${ }^{66}$ Antitubercular therapy augmentation and steroid administration resulted in resolution of symptoms 4 weeks later. ${ }^{66}$ In another patient, MR imaging and CT were used to detect TB spondylitis and paraspinal and epidural extension in a patient with paradoxical TB-IRIS who developed back and leg pain 2 months after antiretroviral therapy initiation. ${ }^{66}$ Corticosteroids were added as well as anti-inflammatory medications with improvement in neurologic symptoms. ${ }^{66}$

In a separate work by Marais et al, ${ }^{67}$ the heightened inflammatory response against the $M$ tuberculosis antigens in patients with paradoxical TB-IRIS was reported to result in new or worsening radiologic findings, including tuberculous abscesses, tuberculomata, meningitis, and hydrocephalus. One such HIV + patient under medical therapy for TB meningitis developed new neurologic symptoms 7 days following HAART initiation, and a contrast CT scan showed leptomeningeal enhancement, hydrocephalus, and ring-enhancing lesions thought to be tuberculomata. ${ }^{67}$ Neurologic examination findings returned to normal after continuation of antituberculous therapy, antiretroviral therapy, and steroids. ${ }^{67}$

While reduced meningeal enhancement and obstructive hydrocephalus caused by a dampened inflammatory response in $\mathrm{HIV}+$ patients with severe immunosuppression without antiretroviral therapy has been reported by some investigators to be a differentiating point from CNS TB with IRIS, nevertheless most authors in fact have stressed the overlap in imaging findings. ${ }^{67}$ Marais et $\mathrm{al}^{67}$ pointed out that the frequency of radiologic findings reported in the literature, including basal meningeal enhancement, hydrocephalus, tuberculomata, and infarcts, was quite similar between HIV-infected with or without IRIS and HIV-negative patients with tuberculous meningitis, as illustrated in Fig 5. Meningeal enhancement and contrast-enhancing lesions, for example, were seen in every group.

It is evident, then, when comparing the 3 different cohorts: namely patients with CNS TB without HIV coinfection with those with CNS TB with HIV co-infection with those with CNS TB with HIV co-infection and IRIS, that there are many similarities, with numerous overlapping clinical and imaging findings. Untreated tuberculous meningitis, even without coinfection with HIV, is a disabling disease associated with a poor outcome in more than half of patients (including morbidity and mortality). ${ }^{68}$ By causing a necrotizing granulomatous inflammatory response, TB exudates can obstruct the CSF pathways, causing hydrocephalus, and can lead to adhesions, cranial nerve palsies, and infarctions from an obliterative arteritis of both large and small vessels (which can be intensified with antituberculous therapy). ${ }^{68}$ Tuberculomas can also develop in $\leq 74 \%$ of patients from coalescence of the granulomas. ${ }^{68}$ In 1 study of 43 patients with TB meningitis, whose neuroimaging showed leptomeningeal enhancement, hydrocephalus, tuberculomas, and/or infarcts, improved survival but not severe disability was seen only in the 24 patients who received steroids. ${ }^{68}$ Decreasing hydrocephalus and infarct prevention were noted in these 24 patients. ${ }^{68}$

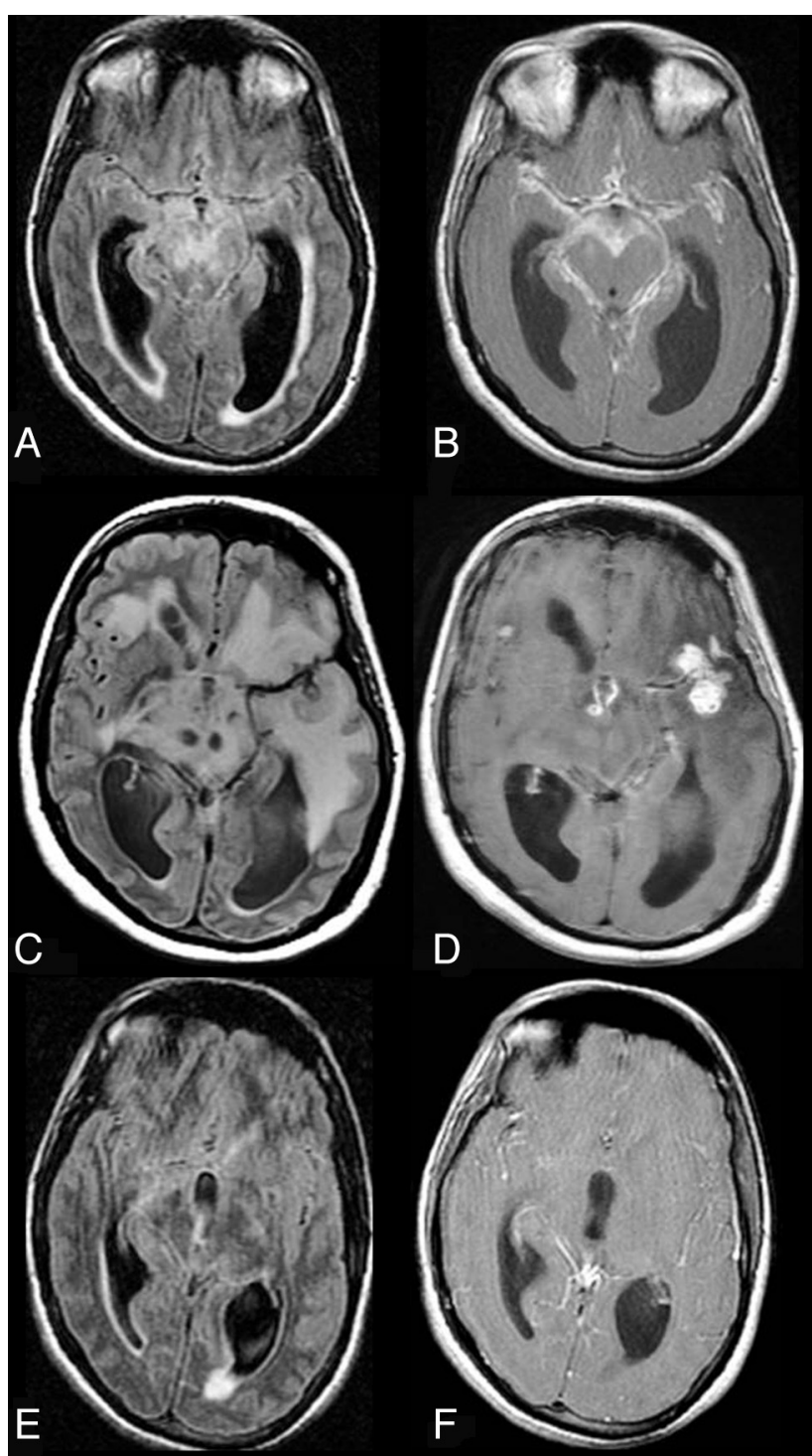

FIG 5. CNS TB. A 28-year-old man presented with headache and vomiting for 3-4 weeks and meningeal irritation. The first MR imaging reveals, on axial FLAIR $(A)$, a communicating hydrocephalus with transexudation of CSF and, on contrast axial TIWI (B), avid and diffuse enhancement of the leptomeningeal spaces compatible with meningitis. On routine CSF examination, PCR for TB was consistent with TB meningitis. The patient was kept on antitubercular treatment and showed clinical signs of deterioration after 8 weeks of therapy. Follow-up axial FLAIR (C) and contrast TIWI (D) reveal a marked increase in edema, evidenced by the diffuse hypertense signal in the brain parenchyma $(C)$, persistent hydrocephalus, and new enhancing parenchymal lesions $(D)$, compatible with tuberculomas. However, the basal meningeal enhancement showed improvement. After 6 months of therapy ( $E$ and $F$ ), there is MR imaging improvement associated with clinical improvement. Axial FLAIR $(E)$ reveals a marked decrease in edema and in the ventricular size and resolution of the tuberculomas. While this patient was not HIV-infected and was not on HAART, the imaging appearance is similar to that seen in CNS TB-IRIS.

Similarly, CNS tuberculosis with HIV coinfection also has a very high mortality rate, and imaging findings can resemble those in patients without HIV coinfection as well as those in patients with neurologic IRIS and HIV. ${ }^{69}$ For example, in a study of 25 $\mathrm{HIV}+$ patients without IRIS, CT or MR imaging showed enhancing parenchymal lesions in $44 \%$ ( 6 with tuberculomata and 5 with tuberculous abscesses), meningeal enhancement in 36\%, infarc- 
tion in 36\%, and communicating hydrocephalus in 32\%. ${ }^{69}$ It was only the TB abscesses that were noted to be more frequent in this HIV + population than in the non-HIV cohort. ${ }^{69}$ Finally, although some investigators have suggested that meningeal enhancement and a communicating hydrocephalus in a patient with antitubercular and antiretroviral therapy should point to a diagnosis of TB-CNS-IRIS, ${ }^{19,30}$ these imaging findings are not exclusive to this group of patients, even if some authors have reported them more commonly in the IRIS cohort.

\section{Parasites}

Toxoplasma Encephalitis-IRIS. While toxoplasma encephalitisIRIS has been described, it is much less frequently seen than PMLIRIS and cryptococcal CNS-IRIS. ${ }^{70-76}$ In a case reported by Tsambiras et al, ${ }^{70}$ neurologic symptoms developed 3 weeks after initiation of HAART in a patient newly diagnosed with HIV whose CD4 count was increasing and whose viral load was decreasing and who had a positive serum antibody assay for Toxoplasma IgG 1 month before hospitalization. MR imaging revealed multiple enhancing nodules without edema due to toxoplasma encephalitis, which responded to medical therapy for this parasitic infection. ${ }^{70}$ In another investigation, toxoplasmosis was documented in 5 patients within 15 months of HAART initiation, ${ }^{70}$ while Rodriguez et $\mathrm{al}^{75}$ reported that toxoplasmosis was the opportunistic infection that occurred in 9 instances of opportunistic infection developing in a cohort of 247 patients treated with HAART. When one compares the imaging appearance in HIV+ patients with toxoplasma encephalitis without IRIS with those with IRIS, the similarities stand out. Focal enhancing parenchymal mass lesions with edema are seen in both groups, with only 1 report ${ }^{74}$ suggesting an uncharacteristic finding of a speckled enhancement pattern in addition to the more typical focal ring enhancement. The clinical scenario must be the differentiating point then.

When CNS-IRIS is seen in association with the parasite Toxoplasma gondii, a granulomatous inflammatory response is induced, similar to the response evoked by fungal and mycobacterial infections. ${ }^{70}$ This pathologic response is different from the reaction induced by viruses such as CMV, HIV, or the JC virus, which is characterized by cytotoxic CD8 T lymphocytic perivascular infiltration associated with CD68 macrophage infiltration. ${ }^{6,22,59,70}$ It is evident then that the pathologic response induced by CNS-IRIS varies with the organism type. ${ }^{70}$

\section{CNS-IRIS WITHOUT ORGANISMS \\ Neuro-IRIS without Coinfection}

As alluded to earlier in this article, following HAART an exaggerated immune response can occur to an antigenic stimulus when the immune system is being restored even in the absence of an infectious agent and even at long time intervals after the initiation of HAART. In a recent case report, neurologic symptoms developed in an HIV + patient a full 2 years after HAART initiation. ${ }^{77}$ During the ensuing 2 years, the patient's clinical course was characterized by a chronic relapsing and remitting meningitis with a lymphocytic pleocytosis without detectable organisms. ${ }^{77} \mathrm{MR}$ imaging at various time intervals (Fig 6) showed leptomeningeal enhancement (which did not persist)

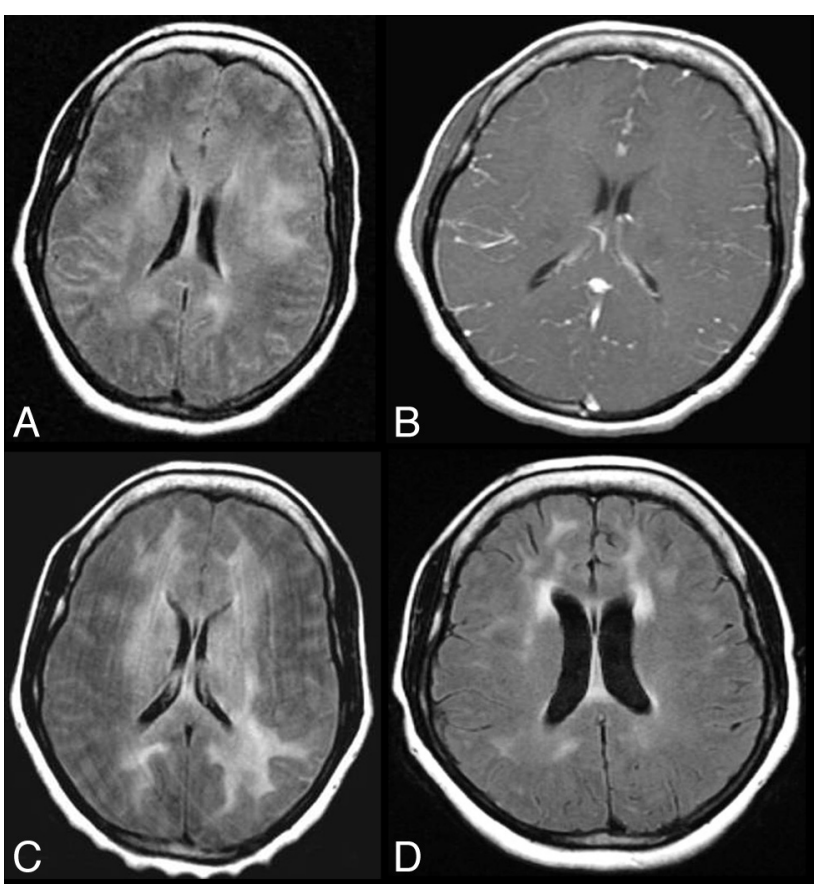

FIG 6. Chronic CNS-IRIS without coinfection. Two years following the institution of HAART, this HIV-positive patient developed a chronic relapsing and remitting meningitis without detectable organisms. Initial MR imaging on axial FLAIR $(A)$ shows bilateral diffuse deep and subcortical white matter hyperintensities with no atrophy and with high signal in the subarachnoid spaces, while on axial contrast TTWI (B), leptomeningeal enhancement is seen. Minimal brain stem high FLAIR signal is also demonstrated (not shown). Two years later at the time of greatest neurologic impairment, axial FLAIR MR imaging (C) reveals mild mass effect and progression of the supratentorial and brain stem lesions. No organism was detected by CSF analysis. Following brain biopsy and steroid administration, there was symptom resolution. A follow-up MR imaging on axial FLAIR $(D)$ reveals resolution of mass effect and a decrease in white matter lesions. Figures $A, C$, and $D$ reproduced with permission from Costello et al. ${ }^{77}$

as well as white matter lesions, consistent with a meningoencephalitis from an inflammatory reaction. ${ }^{77}$ Extension of brain stem lesions, worsening of the nonenhancing supratentorial white matter lesions, and mass effect manifested by sulcal and ventricular compression were all seen on the MR imaging 4 years after HAART initiation when the patient had her most severe neurologic decline. ${ }^{77}$ It was postulated that the patient's recurrent symptoms were related to transient and intermittent leaks of viruses, namely HIV and Epstein-Barr virus, into the CSF, resulting in an antigenic intrathecal stimulus and an immune activation with an IRIS response. ${ }^{77}$ Therefore, following brain biopsy, steroids were administered, which led to symptom resolution. On MR imaging, a reduction of the FLAIR/T2 hyperintense signal abnormalities in the supratentorial white matter, resolution of mass effect, and resolution of the brain stem signal changes were visualized in this patient with chronic CNS-IRIS. ${ }^{77}$

\section{Tumefactive Inflammatory CNS Demyelinating Disease and Fulminating Leukoencephalopathy}

Some authors have reported that IRIS when occurring in the absence of opportunistic infections can rarely cause, early on, a severe autoimmune phenomenon to either the CNS or to the peripheral nervous 
system, which may necessitate treatment with immunomodulatory therapy, osmotherapy, and steroids. ${ }^{78,79}$ In an article by Lindzen et $\mathrm{al}^{78}$ a progressive tumefactive inflammatory CNS demyelinating disease resembling the Marburg variant of multiple sclerosis was attributed to an autoimmune response triggered by HAART in a patient with AIDS. This heightened immune reaction to the release of self-antigens was thought to have an underlying genetic predisposition. ${ }^{78}$ In another investigation by Oelschlaeger et al, ${ }^{79}$ a severe autoimmune response was found at postmortem examination in the brain of an HIV + patient who had a fulminating clinical course after a change in HAART and a very rapid immune response. IRIS in this patient led to a marked leukoencephalopathy, cerebral edema, and brain herniation. ${ }^{79} \mathrm{MR}$ imaging showed parietal-occipital areas of increased T2 signal and avid enhancement in the perivascular spaces. $^{79}$

At postmortem examination, these perivascular spaces were found to be infiltrated by CD8 + lymphocytes, the cause of the enhanced inflammatory response, while the brain parenchyma showed only reactive astrocytes in the white matter. ${ }^{79}$ The authors postulated that the rapid recovery of circulating CD4+ cells following HAART could induce IRIS by triggering a CD8 + lymphocytic T-cell response against antigens, leading to leptomeningitis, vasculitis, or cerebritis. ${ }^{79}$ When that response is aimed against the peripheral nervous system, a peripheral nerve inflammatory demyelinating disease may develop. ${ }^{79}$ A Guillain-Barré syndrome occurring after HAART initiation and linked to immune reconstitution with proliferation of Tcells may then occur. ${ }^{38,79}$ According to a case report by Teo et al, ${ }^{38}$ the cause of Guillain-Barré syndrome in an HIV+ patient treated with antiretroviral therapy was a demyelination, which developed when activated T-cells caused a cytokine release in the endoneurium of the peripheral nerves as they interacted with the viral or bacterial epitopes on Schwann cells.

\section{SUMMARY}

The inflammatory response evoked in the HIV-infected patient following HAART initiation, while potentially beneficial, may cause, if overexuberant, a clinical deterioration necessitating a change in medical management. It is important to recognize this condition then. In the CNS, the diagnosis of IRIS, while often elusive, can be suggested either by imaging findings different from those seen in the pre-HAART era, such as in VZV meningitis, or by abnormalities typical of an inflammatory response, even if these are similar to the pre-HAART appearance, such as in mycobacterial infection and Toxoplasma encephalitis.

Disclosures: Majda Thurnher—UNRELATED: Royalties: Amirsys. David Clifford—UNRELATED: Consultancy: All <\$10,000 annually: Biogen Idec, Genentech, Millennium, Genzyme, Bristol Myers Squibb, Pfizer, Janssen, Expert Testimony: Biogen Idec, Comments: European Medicines Agency (EMA) discussion of natalizumab, Payment for Development of Educational Presentations: Millennium, payment for the teaching video on the examination for PML; Genentech, payment for the teaching video on PML diagnosis, Other: Millennium, Independent Adjudication Committee, Genzyme, Data Monitoring Committee, Chair; Genentech, Panel of Experts, Translational Immunology Consultant; Pfizer, Data Safety Monitoring Committee (DSMB).

\section{REFERENCES}

1. Johnson T, Nath A. Neurological complications of immune reconstitution in HIV-infected populations. Ann N Y Acad Sci 2010;1184:106-20

2. Shelburne SA, Visnegarwala F, Darcourt J, et al. Incidence and risk factors for immune reconstitution inflammatory syndrome during highly active antiretroviral therapy. AIDS 2005;19:399-406

3. Shelburne SA III, Hamill R. The immune reconstitution inflammatory syndrome. AIDS Rev 2003;5:67-79

4. Shelburne SA, Montes M, Hamill RJ. Immune reconstitution inflammatory syndrome: more answers, more questions. J Antimicrob Chemother 2006;57:167-70

5. Muller M, Wandel S, Colebunders R, et al. Immune reconstitution inflammatory syndrome in patients starting antiretroviral therapy for HIV infection: a systematic review and meta-analysis. Lancet Infect Dis 2010;10:251-61

6. Vendrely A, Bienvenu B, Gasnault J, et al. Fulminant inflammatory leukoencephalopathy associated with HAART-induced immune restoration in AIDS-related progressive multifocal leukoencephalopathy. Acta Neuropathol 2005;109:449-55

7. Shelburne SA III, Hamill RJ, Rodriquez-Barradas MC, et al. Immune reconstitution inflammatory syndrome: emergence of a unique syndrome during highly active antiretroviral therapy. Medicine (Baltimore) 2002;81:213-27

8. Bonham S, Meya DB, Bohjanen PR, et al. Biomarkers of HIV immune reconstitution inflammatory syndrome. Biomark Med 2008;2:349-61

9. Manabe Y, Campbell JD, Syndor E, et al. Immune reconstitution inflammatory syndrome: risk factors and treatment applications. $J$ Acquir Immune Defic Syndr 2007;46:456-62

10. Grant PM, Komarow L, Andersen J, et al. Risk factor analyses for immune reconstitution inflammatory syndrome in a randomized study of early vs. deferred ART during an opportunistic infection. PLOS ONE 2010; 5:e11416

11. Dhasmana DJ, Dheda K, Ravn P, et al. Immune reconstitution inflammatory syndrome in HIV-infected patients receiving antiretroviral therapy: pathogenesis, clinical manifestations and management. Drugs 2008;68:191-208

12. French MA. Disorders of immune reconstitution in patients with HIV infection responding to antiretroviral therapy. Curr HIV/AIDS Rep 2007;4:16-21

13. French MA, Lenzo N, John M, et al. Immune restoration disease after the treatment of immunodeficient HIV-infected patients with highly active antiretroviral therapy. HIV Med 2000;1:107-15

14. French MA. Immune reconstitution inflammatory syndrome: a reappraisal. Clin Infect Dis 2009;48:101-07

15. Tan K, Roda R, Ostrow L, et al. PML-IRIS in patients with HIV infection. Clinical manifestations and treatment with steroids. Neurology 2009;72:1458-64

16. Berkeley JL, Nath A, Pardo CA. Fatal immune reconstitution inflammatory syndrome with human immunodeficiency virus infection and Candida meningitis: case report and review of the literature. J Neurovirol 2008;14:267-76

17. Navas E, Martin-Davilla P, Moreno L, et al. Paradoxical reactions of tuberculosis in patients with the acquired immunodeficiency syndrome who are treated with highly active antiretroviral therapy. Arch Inn Med 2002;162:97-99

18. Murdoch DM, Venter WDF, Van Rie A, et al. Immune reconstitution inflammatory syndrome (IRIS): review of common infectious manifestations and treatment options. AIDS Res Ther 2007;4:1-10

19. Riedel DJ, Pardo CA, McArthur J, et al. Therapy insight: CNS manifestations of HIV-associated immune reconstitution inflammatory syndrome. Nat Clin Prac Neurol 2006;2:557-65

20. Bicanic T, Meintjes G, Rebe K, et al. Immune reconstitution inflammatory syndrome in HIV-associated cryptococcal meningitis: a prospective study. J Acquir Immune Defic Syndr 2009;51:130-34

21. McCombe JA, Auer RN, Maingat FG, et al. Neurologic immune reconstitution inflammatory syndrome in HIV/AIDS: outcome and epidemiology. Neurology 2009;72:835-41

22. Venkataramana A, Pardo CA, McArthur JC, et al. Immune reconstitution inflammatory syndrome in the CNS of HIV-infected patients. Neurology 2006;67:383-88

23. Gray F, Bazille C, Biassette-Adle, et al. Central nervous system im- 
mune reconstitution disease in acquired immunodeficiency syndrome patients receiving highly active antiretroviral treatment. J Neurovirol 2005;11:16-22

24. van der Ven AJ, van Oostenbrugge RJ, Kubat B, et al. Cerebral vasculitis after initiation antiretroviral therapy. AIDS 2002;16:2362-64

25. Murdoch DM, Venter WDF, Feldman C, et al. Incidence and risk factors for the immune reconstitution inflammatory syndrome in HIV patients in South Africa: a prospective study. AIDS 2008;22:601-10

26. Robertson J, Meier M, Wall J, et al. Immune reconstitution syndrome in HIV: validating a case definition and identifying clinical predictors in persons initiating antiretroviral therapy. Clin Infec Dis 2006;42:1639-46

27. Stoll M, Schmidt RE. Immune restoration inflammatory syndromes: apparently paradoxical clinical events after the initiation of HAART. Curr HIV/AIDS Rep 2004;1:122-27

28. Singer EJ, Sueiras-Valdes M, Commins D, et al. Neurologic presentations of AIDS. Neurol Clin 2010;28:253-75

29. Sexton DJ, Pien BC, et al. Immune reconstitution inflammatory syndrome. UpToDate. Available at http://www.uptodate.com. Accessed January 31,2012

30. McCarthy M, Nath A. Neurologic consequences of the immune reconstitution inflammatory syndrome (IRIS). Curr Neurol Neurosci Rep 2010;10:467-75

31. McArthur JC, Steiner J, Sacktor N, et al. Human immunodeficiency virus-associated neurocognitive disorders: mind the gap. Ann Neurol 2010;67:699-714

32. DeSimone JA, Pomerantz RJ, Babinchak TJ. Inflammatory reactions in HIV-1-infected persons after initiation of highly active antiretroviral therapy. Ann Intern Med 2000;133:447-54

33. French MA, Mallal SA, Dawkins RL. Zidovudine-induced restoration of cell-mediated immunity to mycobacteria in immunodeficient HIV-infected patients. AIDS 1992;6:1293-97

34. Ohta K, Kishida S. Immune reconstitution inflammatory syndrome in the central nervous system. Brain Nerve 2007;59:1355-62

35. Rushing EJ, Liappis A, Smirniotopoulos JD, et al. Immune reconstitution inflammatory syndrome of the brain: case illustrations of a challenging entity. J Neuropathol 2008;67:819-27

36. Nath A, Geiger J. Neurobiological aspects of human immunodeficiency virus infection: neurotoxic mechanisms. Prog Neurobiol 1998;54:19-33

37. Navdeesh S, McCutchan JA. Unmasking of PML by HAART: unusual clinical features and the role of IRIS. J Neuroimmunol 2010;219:100-04

38. Teo EC, Azwra A, Jones R, et al. Guillain-Barre syndrome following immune reconstitution after antiretroviral therapy for primary HIV infection. J HIV Ther 2007;12:62-63

39. Anthony IC, Bell JE. Neuropathological findings associated with long-term HAART. In: Paul RH, Sacktor NC, Valcour V, et al, eds. HIV and the Brain. New Challenges in the Modern Era. New York: Humana Press; 2009:29-47

40. Bazille GF, Biassette MH, Moulignier A, et al. Central nervous system immune reconstitution disease in acquired immunodeficiency syndrome patients receiving highly active antiretroviral treatment. J Neurovirol 2005;3:16-22

41. Ratnam I, Chiu C, Kandala NB, et al. Incidence and risk factors for immune reconstitution inflammatory syndrome in an ethnically diverse HIV type 1-infected cohort. Clin Infect Dis 2006;42:418-27

42. Huruy K, Andargachew M, Getahun M, et al. Immune reconstitution inflammatory syndrome among HIV/AIDS patients during highly active antiretroviral therapy in Addis Ababa, Ethiopia. J Infect Dis 2008;61:205-09

43. Gray F, Chrétien F, Vallat-Decouvelaere AV, et al. The changing pattern of HIV neuropathology in the HAART era. J Neuropathol Exp Neurol 2003;62:429-40

44. Price P, Morahan G, Huang D, et al. Polymorphisms in cytokine genes define subpopulations of HIV-1 patients who experienced immune restoration diseases. AIDS 2002;16:2043-47
45. Wang ME, Castillo ME, Silvia M, et al. Immune reconstitution inflammatory syndrome in human immunodeficiency virus-infected children in Peru. Pediatr Infect Dis J 2009;28:900-03

46. Kilborn T, Zampoli M. Immune reconstitution inflammatory syndrome after initiating highly active antiretroviral therapy in HIVinfected children. Pediatr Radiol 2009;39:569-74

47. Newsome SD, Nath A. Varicella-zoster virus vasculopathy and central nervous system immune reconstitution inflammatory syndrome with human immunodeficiency virus infection treated with steroids. J Neurovirol 2009;15:288-91

48. Nagel MA, Cohrs RJ, Mahalingam R, et al. The varicella zoster vasculopathies: clinical, CSF, imaging and virologic features. $\mathrm{Neu}$ rology 2008;70:853-59

49. Nanavati KI, Post MJ, Nagornaya N. Value of MRI in the diagnosis of clinically elusive herpes zoster myelitis in HIV-positive patients. In: Proceedings of the American Society of Spine Radiology Annual Symposium, Miami Beach, Florida. February 18-22, 2009

50. Espinosa E, Pena-Jimenez A, Ormsby CE, et al. Later onset of herpes zoster-associated immune reconstitution inflammatory syndrome. HIV Med 2009;10:454-57

51. Steininger C, Puchhammer-Stocki E, Popow-Kraupp. Cytomegalovirus disease in the era of highly active antiretroviral therapy (HAART). J Clin Virol 2006;37:1-9

52. Karavellas MP, Lowder CY, MacDonald C, et al. Immune recovery vitritis associated with inactive cytomegalovirus retinitis: a new syndrome. Arch Ophthalmol 1998;116:169-75

53. Jacobson MA, Zegans, Pavan PR, et al. Cytomegalovirus retinitis after initiation of highly active antiretroviral therapy. Lancet 1997;349:1443-45

54. Anderson AM, Mosunjac MB, Corey AS, et al. Simultaneous typical and extraordinary imaging findings of AIDS-associated cytomegalovirus encephalitis. J Neurol Sci 2011;307:174-77

55. Anderson AM, Fountain JA, Green SB, et al. Human immunodeficiency virus-associated cytomegalovirus infection with multiple small vessel cerebral infarcts in the setting of early immune reconstitution. J Neurovirol 2010;16:179-84

56. Morgello S. Cho ES, Nielsen S, et al. Cytomegalovirus encephalitis in patients with the acquired immunodeficiency syndrome: an autopsy of 30 cases and a review of the literature. Hum Pathol 1987;18:289-97

57. Melica G, Brugieres P, Lascaux AS, et al. Primary vasculitis of the central nervous system in patients infected with HIV-1 in the HAART era. J Med Virol 2009;81:578-81

58. Thurnher MM, Schindler EG, Thurnher SA, et al. Highly active antiretroviral therapy for patients with AIDS dementia complex: effect on MRI imaging findings and clinical course. AJNR Am J Neuroradiol 2000;21:670-78

59. Miller RF, Issacson PG, Hall-Craggs M, et al. Cerebral CD8 lymphocytosis in HIV-1 infected patients with immune restoration induced by HAART. Acta Neuropathol 2004;108:17-23

60. Langford TD, Letendre SL, Marcotte TD, et al. Severe demyelinating leukoencephalopathy in AIDS patients on antiretroviral therapy. AIDS 2002;16:1019-29

61. Tavazzi E, Bargiggia V, Pichiecchio A, et al. HIV-related acute inflammatory leukoencephalopathy of undetermined origin: review of the literature. Int J Immunopathol Pharmacol 2010;23:693-700

62. Kishida S, Ajisawa A. Probable cerebral mycobacterium avium complex-related immune reconstitution inflammatory syndrome in an HIV-infected patient. Intern Med 2008;47:1349-54

63. Mohanty K. Immune reconstitution inflammatory syndrome after initiation of highly active anti-retroviral therapy in HIV/AIDS. Indian J Dermatol Venereol Leprol 2010;76:301-04

64. Murray R, Mallal S, Heath C, et al. Cerebral mycobacterium avium infection in an HIV-infected patient following immune reconstitution and cessation of therapy for disseminated mycobacterium avium complex infection. Eur J Clin Microbiol Infect Dis 2001;20:199

65. Breton G, Duval X, Estellat C, et al. Determinants of immune reconstitution inflammatory syndrome in HIV type 1-infected patients 
with tuberculosis after initiation of antiretroviral therapy. Clin Infect Dis 2004;39:1709-12

66. Pepper DJ, Marais S, Maartens G, et al. Neurologic manifestations of paradoxical tuberculosis-associated immune reconstitution inflammatory syndrome: a case series. Clin Infect Dis 2009;48:e96-107

67. Marais S, Pepper DJ, Marais B, et al. HIV-associated tuberculous meningitis: diagnostic and therapeutic challenges. Tuberculosis ( $E d-$ inb) 2010;90:364-74

68. Thwaites GE, Macmullen-Price J, Tran TH, et al. Serial MRI to determine the effect of dexamethasone on the cerebral pathology of tuberculous meningitis: an observational study. Lancet Neurol 2007;6:230-36

69. Whiteman M, Espinoza L, Post MJ, et al. CNS tuberculosis in HIVinfected patients: clinical and radiographic findings. AJNR Am J Neuroradiol 1995;16:1319-27

70. Tsambiras PE, Larkin JA, Houston SH. Case report: toxoplasma encephalitis after initiation of HAART. AIDS Read 2001;11:615-16

71. Ledergerber B, Egger M, Erard V, et al. AIDS-related opportunistic illnesses occurring after initiation of potent antiretroviral therapy: the Swiss HIV cohort study. JAMA 1999;282:2220-26

72. Balkhair A, Ahamed S, Sankhla D. Unmasking immune reconstitution inflammatory syndrome (IRIS): A report of five cases and review of the literature. Sultan Qaboos Univ Med J 2011;11:95-103
73. Tremont-Lukats IW, Garciarena P, Juarbe R, et al. The immune inflammatory reconstitution syndrome and central nervous system toxoplasmosis. Ann Intern Med 2009;150:656-57

74. Pfeffer G, Prout A, Hooge J, et al. Biopsy-proven immune reconstitution syndrome in a patient with AIDS and cerebral toxoplasmosis. Neurology 2009;73:321-22

75. Rodriguez RR, Sorlano V, Dona C, et al. Opportunistic infections shortly after beginning highly active antiretroviral therapy. Antivir Ther 1998;3:229-31

76. Martin-Blondel G, Alvarez M, Delobel P, et al. Toxoplasmic encephalitis IR IS in HIV-infected patients: a case series and review of the literature. J Neurol Neurosurg Psychiatry 2010;8:691

77. Costello DJ, Gonzalez RG, Frosch MP. Case 18-2011: a 35-year-old HIV-positive woman with headache and altered mental status. N Engl J Med 2011;364:2343-52

78. Lindzen E, Jewells V, Bouldin T, et al. Progressive tumefactive inflammatory central nervous system demyelinating disease in an acquired immunodeficiency syndrome patient treated with highly active antiretroviral therapy. J Neurovirol 2008;14:569-73

79. Oelschlaeger C, Dziewas R, Reichelt D, et al. Severe leukoencephalopathy with fulminant cerebral edema reflecting immune reconstitution inflammatory syndrome during HIV infection: a case report. J Med Case Reports 2010;4:214-16 